\title{
AN AUTOMATED FOCAL POINT POSITIONING AND EMITTANCE MEASUREMENT PROCEDURE FOR THE INTERACTION POINT OF THE SLC *
}

\author{
N. PHINNEY, P. BAMBADE * * and W. KOZANECKI \\ Stanford Linear Accelerator Center, Stanford University, Stanford, CA 94309, USA
}

\author{
W. KOSKA + \\ University of Michigan, Ann Arbor, MI 48109, USA
}

Received 25 August 1989

To achieve maximum luminosity at the SLC, both the electron and positron beams must reach their minimum transverse size within $1 \mathrm{~mm}$ of the longitudinal location where the two bunches collide. This paper describes an automated procedure for positioning the focal point of each beam at this collision point. The technique is based on measurements of the beam size utilizing either secondary emission or bremsstrahlung signals from carbon fibers a few microns in diameter. We have achieved simultaneous and reproducible measurements of the angular spread ( $\sim 200 \mu \mathrm{rad}$ ) and of the optimum beam spot size $-2 \mu \mathrm{m}$ ), which when combined yield measurements of the beam emittance consistent with those obtained using conventional profile monitor techniques.

\section{Introduction}

The Final Focus System (FFS) for the SLAC Linear Collider (SLC) [1] is designed to produce beam spots a few microns in diameter at their waists. To produce maximum luminosity, the longitudinal positions of the horizontal and vertical waists of both beams must coincide with the interaction point (IP). The standard technique for determining waist position uses a fluorescent screen profile monitor to measure beam spot size for a range of focal point positions [2]. However, the resolution of a screen is inadequate to measure spots on the order of a few microns. Instead, we use a fine carbon wire, which allows us to measure beam profiles in one dimension at a time. The focal position of the beam (or, equivalently, the Twiss parameter $\alpha$ ) is varied and the beam size is measured for at least four settings. A parabolic fit to the square of the beam size versus the focal position yields the size of the beam at the waist, the angular divergence, and the distance from the waist to the IP.

In sections 2 and 3 we describe the details of an automated procedure, now used routinely to measure

- Work supported by Department of Energy contracts DEAC03-76SF00515 and DE-AC02-76ER01112.

* Present address. Laboratoire de l'Accélérateur Linéaire, Bat 200, Orsay 91405, France.

+ Present address: Fermi National Accelerator Laboratory, P.O. Box 500, Batavia, IL 60510, USA. the angular divergence and to position the focal point at the IP. Section 4 discusses the generalization of this method to the minimization of dispersion and crossplane coupling [3]. After full first-order optimization of the spot size, the beam waist and the angular divergence are simply related to the emittance and beta function at the IP, providing a measurement of these parameters for simultaneous first- and second-order optimization of the spot size. The concepts and methods developed with this technique are readily adapted to luminosity optimization using the beam-beam interaction $[4,5]$.

\section{Principle of the technique}

The horizontal beam size at the IP, $\sigma_{h}$, can be expressed as a function of the horizontal beam size at the waist, $\sigma_{h}(w)$, the beam angular divergence, $\sigma_{h}^{\prime}$, and the distance from the beam waist in that dimension to the IP, $\left(z_{\mathrm{h}}-z_{\mathrm{IP}}\right)$ :

$\sigma_{\mathrm{h}}^{2}=\sigma_{\mathrm{h}}^{2}(w)+\sigma_{\mathrm{h}}^{\prime 2}\left(z_{\mathrm{h}}-z_{\mathrm{IP}}\right)^{2}$.

Similarly, in the vertical plane,

$\sigma_{\mathrm{v}}^{2}=\sigma_{\mathrm{v}}^{2}(w)+\sigma_{\mathrm{v}}^{\prime 2}\left(z_{\mathrm{v}}-z_{\mathrm{IP}}\right)^{2}$.

These equations form the basis for the waist positioning and emittance measuring technique. As illustrated in fig. 1, the longitudinal position of either the horizontal or the vertical waist of a beam is moved in a series of steps over a range large enough (typically, a few centim- 


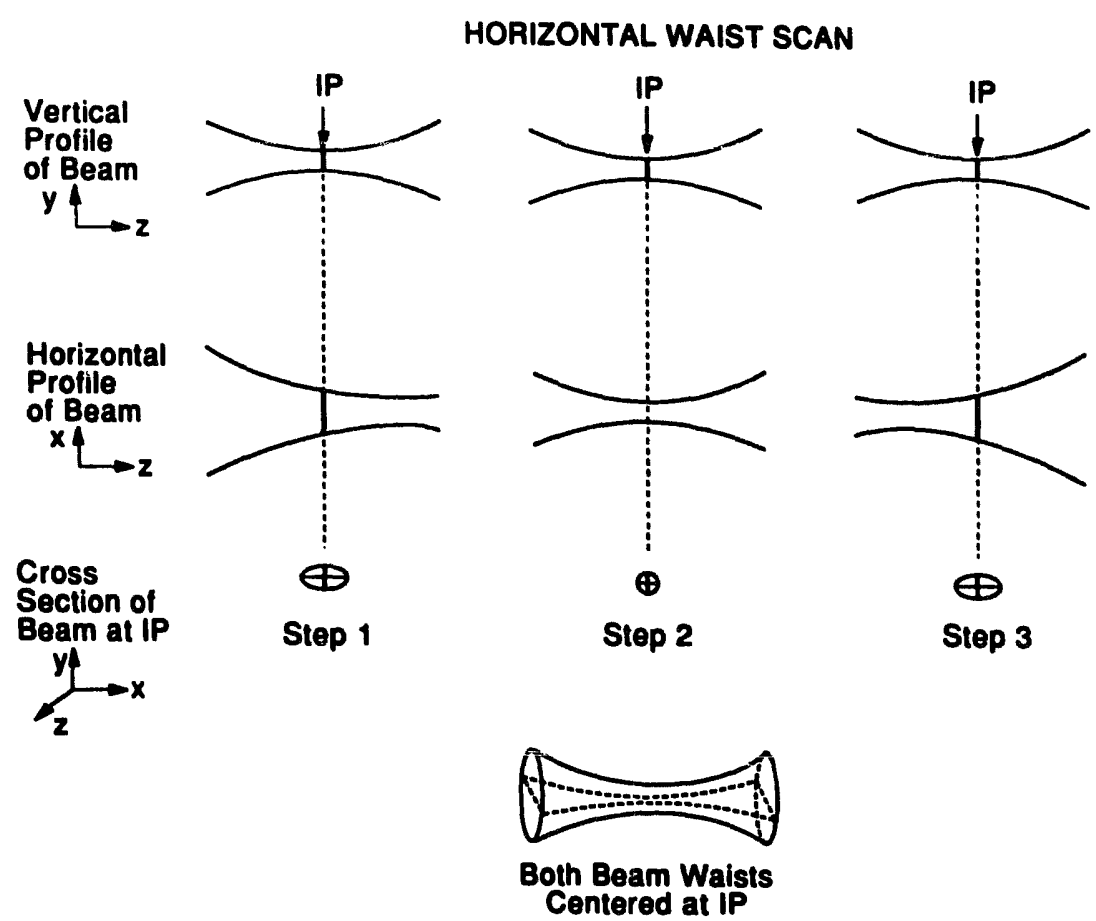

Fig. 1. Schematic representation of a horizontal waist scan. The horizontal waist is moved longitudinally in a series of steps, while the vertical waist is kept fixed. A similar procedure is used for the vertical waist.

eters) to induce a significant variation of the spot size. At each step, the beam size at the IP is measured. Upon completion of such a "waist scan," a parabolic fit of the measured beam sizes squared versus waist position yields the parameters $\sigma(w), \sigma^{\prime}$, and $\left(z-z_{1 P}\right)$. If previous tuning has eliminated dispersion at the IP and removed all cross-plane coupling terms, and if higher-order aberrations are negligible, these parameters can be related to the horizontal emittance $\epsilon$ and to the value of the $\beta$ function at the IP $\left(\beta_{\mathrm{h}}^{*}\right)$ through:

$\sigma_{h}(w)=\sqrt{\epsilon \beta_{h}^{*}}$,

$\sigma_{h}^{\prime}=\sqrt{\epsilon / \beta_{h}^{*}}$,

and similarly in the vertical plane.

\section{Mechanics of a waist scan}

For efficient operation of the SLC, the diagnostics outlined above must be performed as quickly as possible - which led us to automate the procedure. The technique separates into three operations: waist positioning, beam scanning, and beam size measurement.

The hardware utilized is illustrated in fig. 2. The longitudinal position of the beam waist is controlled by the low $\boldsymbol{\beta}$ quadrupoles. A pair of trim windings on the quadrupoles can be adjusted in two orthogonal linear combinations which move either the horizontal or the vertical waist by up to $\pm 5 \%$ of the focal length $( \pm 50$ $\mathrm{cm})$, along the beam direction, to an accuracy of less than $1 \mathrm{~nm}$.

The transverse beam position at the IP is controlled by two sets of magnets: an $x, y$ pair of iron-core dipole magnets, and an $x, y$ pair of air-core dipole magnets. The iron-core magnets have a full range at the IP of about $\pm 1 \mathrm{~mm}$ of transverse motion and are used for large changes in beam position. The air-core magnets have a range of only about $\pm 60 \mu \mathrm{m}$ but can reposition the beam in one interpulse period.

The transverse beam size is measured by sweeping the beam, using one air-core dipole, in a series of 1 or 2 $\boldsymbol{\mu} \mathrm{m}$ steps across wire targets supported by a fork which can be introduced into the center of the beam pipe at the IP [6]. When the beam intercepts the wire, both secondary emission and bremsstrahlung are produced

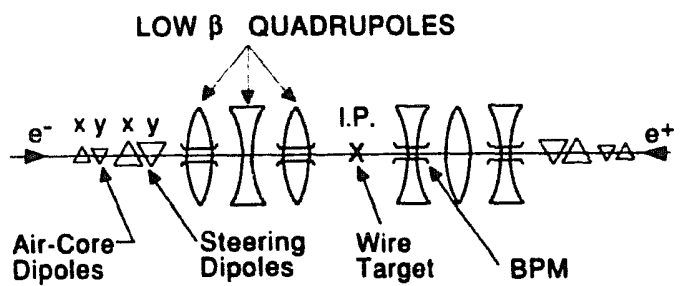

Fig. 2. Beamline components used in performing waist-scans are illustrated. The waist is moved by adjusting the strengths of the low $\beta$ quadrupoles. Beam sizes are measured using fine carbon fibers at the IP. The beam is oved across the fibers with the dipole magnets upstream of the quadrupoles. 
with an intensity proportional to the number of beam particles striking the wire. The flux of bremsstrahlung is measured in a threshold Cherenkov detector located behind the first main bending magnet downstream of the IP [7]. The wires are approximately 2, 3.5 and 15 $\mu \mathrm{m}$ in radius, which allows us to accurately measure a range of rms beam sizes from $2 \mu 1$. to $100 \mu \mathrm{m}$.

Prior to measuring the beam size, the elestron or positron trajectory must be positioned near one of the wires on the wire scanner fork, in the plane ( $x$ or $y$ ) which is to be scanned. This is achieved by performing a single transverse sweep over a range larger than the interwire distance. The operator chooses the starting position for the sweep relative to the current beam position, the step size (typically $5 \mu \mathrm{m}$ ), and the number of data points to acquire (up to 50). These values are entered, using a touch panel screen, into the host computer running the SLC Control Program [8]. The sweep is then initiated. The software calculates the setting of the iron-core steering magnet (fig. 2) required to put the beam at the starting position. It also precalculates the sequence of settings for the air-core steering magnet to step the beam across the wire. If the sweep range exceeds what can be covered by the air-core magnet alone, the software breaks the sweep into the required number of subsweeps, each of which is controlled by the air-core magnet. After each subsweep, the beam is repositioned with the iron-core magnet to coincide with its position at the end of the previous sequence of steps. The control data for each stepping sequence is passed to a microcomputer which controls the magnets and acquires the data. A readback of the magnet current at each position returns the actual position increment of the beam. Upon completion of the sweep, the data is returned to the host computer for processing. Fig. 3 shows the bremsstrahlung signal from a sweep which intercepted three different-sized wires. The distance between the peaks is consistent with the separation between fibers as measured prior to installation, thereby cross-checking the steering magnet calibrations.

Once the approximate relative position of the beam and the selected wire is known, an automated steering

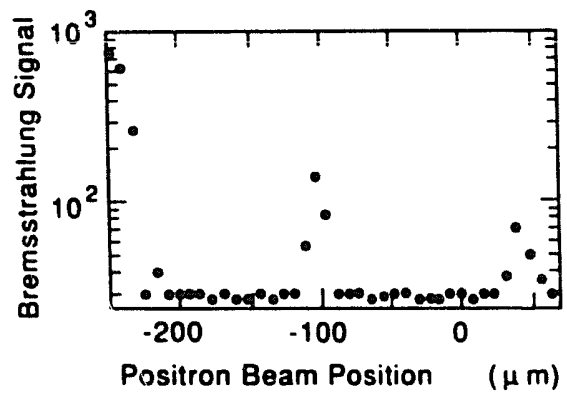

Fig. 3. Results of a single scan of the beam, which intercepted three wires of radius $15,3.5$, and $2 \mu \mathrm{m}$.

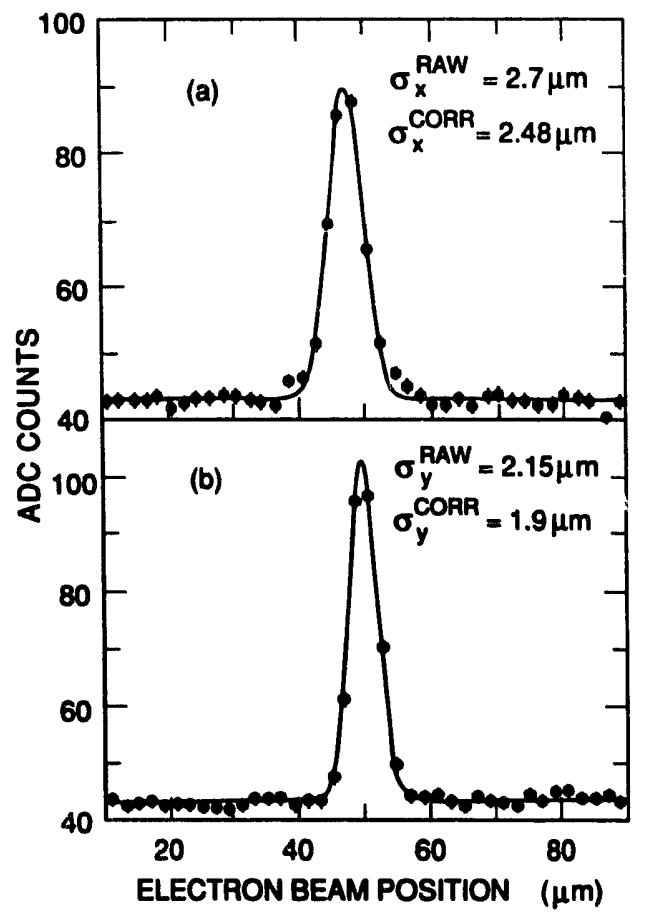

Fig. 4. Signals from $x$ and $y$ sweeps of the $2 \mu \mathrm{m}$ wires showing the Gaussian fits to the data from which the beam size at the IP is extracted. The raw rms beam width is the standard deviation, as determined from this fit. Deconvoluting the contribution from the finite wire size yields the corrected width.

algorithm is used to sweep over a small range about the wire and position the beam a fixed distance (usually $\mathbf{4 0}$ $\mu \mathrm{m})$ from it. Provided that the rms transverse size is not too large, this allows the entire sweep to be performed by the air-core magnets alone. A simple profile measurement can then be completed in a total of about $\mathbf{4 0}$ consecutive beam pulses, or $4 \mathrm{~s}$ with the machine repetition rate limited to $10 \mathrm{~Hz}$. Fig. 4 shows the result of typical sweeps across $2 \mu \mathrm{m}$ horizontal and vertical wires. The curves overlaying the data represent Gaussian fits (on top of a flat background) which are performed automatically after the completion of the sweep, and from which the beam size is extracted. The raw RMS beam width is the standard deviation, as determined from this fit; deconvoluting the contribution from the finite wire radius yields the corrected rms width. In this example, the beam size was measured to be 2.5 by $1.9 \mu \mathrm{m}$ in $x$ and $y$, respectively.

Once the beam is positioned near the wire, the operator selects the range over which to scan the horizontal or vertical waist of the selected beam (typically $\pm 4 \mathrm{~cm}$ about the current waist position, in steps of 2 $\mathrm{cm}$, for a total of five data points). When the scan is initiated, two of the low $\beta$ quadrupoles are adjusted to move the selected waist to the first measurement position, while keeping the waist in the other plane stationary. The beam is then swept across the wire, and a Gaussian fit performed to the resulting measured beam 


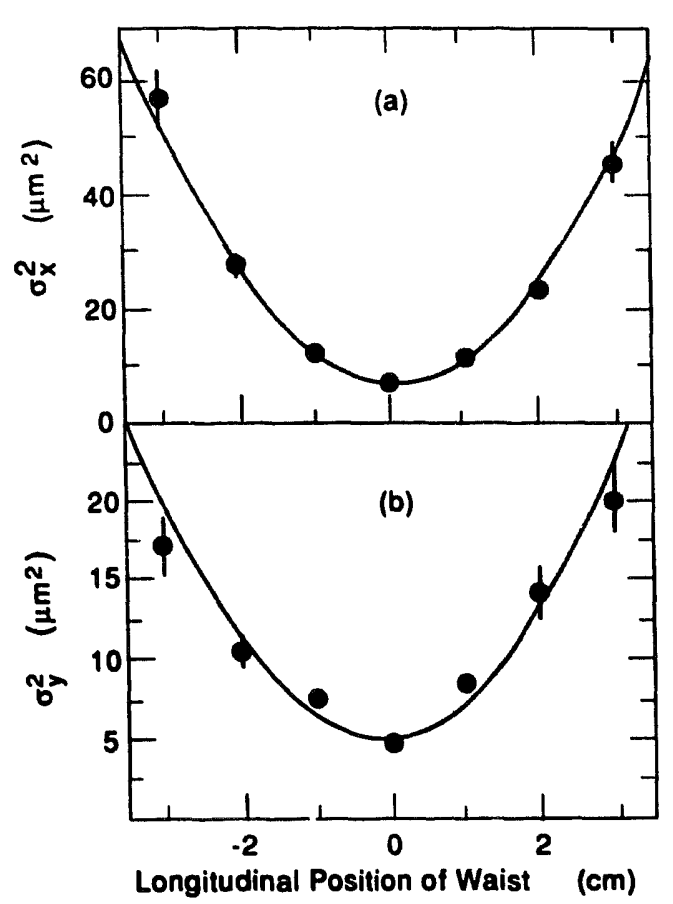

Fig. 5. Results of horizontal and vertical waist-scans of the electron beam. The parabolic fits to the data yielded the emittance, $\beta^{*}$, and the distance from the current waist position to the IP.

profile (Frequently, fluctuations in the linac beam produce bad data during the measurement. If the fitting routine is unable to produce a good Gaussian fit, the procedure automatically repeats the measurement; this increases the success rate of the scans). The waist is then automatically moved to the next position and another sweep made across the wire. This sequence continues until the entire range has been stepped through. At this point, the square of the beam widths extracted from the Gaussian fits are automatically plotted versus waist position, and a parabolic fit is performed. As discussed above, the results of the fit provide the minimum beam size, the angular divergence and the distance from the current waist position to the IP. The operator is then prompted as to whether the waist should be moved to the IP. This procedure can be accomplished in about two minutes under stable beam conditions.

The waist in the other plane is then positioned in the same way. Fig. 5 shows the result of horizontal and vertical waist-scans performed on the electron beam. The result of the fits indicates that the angular divergence of the beam was $220 \pm 4$ and $134 \pm 13 \mu \mathrm{rad}$ in $x$ and $y$, respectively. The waist sizes were $2.6 \pm 0.1$ and $2.3 \pm 0.1 \mu \mathrm{m}$, respectively. The waist scans indicate that the $x$-waist is $1.0 \pm 0.2 \mathrm{~mm}$ and the $y$-waist is $1.3 \pm 1.6$ $\mathrm{mm}$ from the IP, so in this example the waists coincide with the IP. If this were not the case, these values would be used to calculate the settings of the quadrupoles to position the waists at the IP. After these corrections are implemented, the measurement can be repeated with finer steps to improve the accuracy of the waist positioning, if desired.

All measurements are written to the SLC de:a base, from where they are subsequently copied into a history buffer. This provides a means of tracking variations in beam parameters at the IP and of correlating them with changes in other machine parameters.

\section{Extensions of the technique to full first onder optical optimization at the IP}

Several other tuning and diagnostic procedures rely on measurements of the beam size at the IP as a function of the setting of some optical element. These include: elimination of cross-plane coupling through the adjustment of skew quadrupoles; measurement of dispersion at the IP by varying the beam energy and recording the corresponding shift in the centroid of the beam profile; cancellation of residual dispersion by minimizing spot size through the use of closed, dispersion-generating trajectory bumps in the chromatic correction section of the FFS; and measurement of the residual chromaticity as a function of the strength of the final focus sextupoles. The automated operational techniques developed for waist scanning have been adapted to these measurements. In each case, the appropriate optical elements are automatically scanned through a range specified by the operator and the beam size measured at each setting. Upon completion of the scan, the data is analyzed on-line and corrections applied as necessary. The technique has been further extended to use beam size measurements based on the beam-beam interaction in place of the wire-scanner measurements [4].

\section{Measurement of emittance and $\beta^{*}$}

To the extent that all first-order optical corrections have been applied, and that higher-order aberrations remain negligible, the beam size at the waist and the angular divergence provide a measurement of the emittance and of $\beta^{*}$, as described in section 2. For a given emittance, optimum luminosity is achieved when the decrease of the linear spot size with decreasing $\beta^{*}$ is roughly balanced by the concomitant rise in higher-order aberrations [3]. The ability to first measure, and then adjust, the betatron size at the IP is therefore central to the luminosity optimization of the collider.

Table 1 illustrates the results of one such iteration of the betatron matching procedure, which aimed at reducing $\beta^{*}$ by a factor of about two. Column 1 shows the initial measured values of the beam size at the waist and 
Table 1

Results of betatron-matching at the SLC IP.

\begin{tabular}{lccc}
\hline & $\begin{array}{l}\text { Measured } \\
\text { initial } \beta^{*} \\
\text { configuration }\end{array}$ & $\begin{array}{l}\text { Measured } \\
\text { final } \beta^{*} \\
\text { configuration }\end{array}$ & $\begin{array}{l}\text { Design } \\
\text { configuration }\end{array}$ \\
\hline$\sigma_{h}(w)[\mu \mathrm{m}]$ & $3.6^{\mathrm{a})}$ & $2.4^{\mathrm{a})}$ & $2.2^{\mathrm{c})}$ \\
$\sigma_{\mathrm{h}}^{\prime}[\mu \mathrm{rad}]$ & 139 & 212 & 240 \\
$\epsilon_{\mathrm{h}}[\mathrm{m}-\mathrm{rad}]$ & $5.0 \times 10^{-10}$ & $5.1 \times 10^{-10}$ & $4.3 \times 10^{-10}$ \\
$\beta_{\mathrm{h}}^{*}[\mathrm{~mm}]$ & 26 & 11 & $7.5^{\mathrm{b})}$ \\
$\sigma_{\mathrm{v}}(w)[\mu \mathrm{m}]$ & $2.2^{\mathrm{a})}$ & $2.1^{\mathrm{a})}$ & $1.9^{\mathrm{c})}$ \\
$\sigma_{\mathrm{v}}^{\prime}[\mu \mathrm{rad}]$ & 120 & 140 & 240 \\
$\epsilon_{\mathrm{v}}[\mathrm{m}-\mathrm{rad}]$ & $2.6 \times 10^{-10}$ & $2.9 \times 10^{-10}$ & $4.3 \times 10^{-10}$ \\
$\beta_{v}^{*}[\mathrm{~mm}]$ & 18 & 15 & $7.5^{\mathrm{b}}$ \\
\hline
\end{tabular}

a) Corrected for wire resolution.

b) First-order optics only.

c) Includes residual higher-order aberrations.

of the angular divergence of the electron beam, as well as the corresponding estimates of the emittance and of $\beta^{*}$. These values are consistent with emittance measurements performed at the end of the linac. These numbers were used to translate [3] the desired changes in betatron demagnification into new quadrupole settings in the upper transformer of the FFS beamline. Column 2 shows the measured values of the same parameters after implementing these changes. Note that while the measured emittances have remained invariant, as expected, the spot size and the angular divergence have changed (in equal proportions) in opposite directions. Even though the betatron matching procedure achieved only part of the desired demagnification change, the resulting spot sizes (column 2) exceed by less than 15\% the SLC design specifications (column 3).

\section{Summary}

We have combined transverse beam profile measurements, using a wire scanner technique, with automatic stepping of optical beamline elements, to perform a full first-order optimization of micron-sized beams at the interaction point of the SLC. Emittance measurements at the IP are performed rouunely as part of this procedure and yield results consistent with those obtained by conventional techniques further upstream in the accelerator. The measured values of the transverse spot sizes and of the emittance at the collision point were found within $20 \%$ of the design specifications of the collider. The techniques described in this paper and in its companion publications [3-5] form the basis for a more general optimization method, relying on the beam-beam interaction itself, to measure beam sizes. This latter approach should be applicable, at least in its principle, to future linear colliders.

\section{Acknowledgements}

We would like to acknowledge the many contributions to this work by the SLC staff and the Final Fons commissioning group; and, in particular, that of $\mathrm{J}$. Bogart, M. Flores, and A. Gromme for their work on the control software for this procedure.

\section{References}

[1] See, e.g., W. Kozanecki, Proc. 8th Int. Conf. on Physics in Collision, Capri, Italy (1988); SLAC-PUB-4859 (1989).

[2] M.C. Ross et al., Automated Emittance Measurements in the SLC, Washington PAC 1987;725; SLAC-PUB-4278 (1987).

[3] P. Bambade et al., Operational Experience with Optical Matching in the SLC Final Focus System, to be published in Nucl. Instr. and Meth.; SLAC-PUB-4776 (1989).

[4] W. Koska et al., Nucl. Instr. and Meth. A286 (1990) 32; SLAC-PUB-4919 (1989).

[5] E. Gero et al., Beamstrahlung as an Optics Tuning Tool at the SLC IP, Proc. IEEE Particle Accelerator Conf., Chicago, IL (1989); SLAC-PUB-4930 (1989).

[6] G. Bowden et al., Nucl. Instr. and Meth. A278 (1989) 664.

[7] G. Bonvicini et al., Nucl. Instr. and Meth. A277 (1989) 297.

[8] N. Phinney, Proc. IEEE Particle Accelerator Conf. (1985) 2117. 